\title{
TOPOLOGICAL HOCHSCHILD HOMOLOGY AND THE CYCLIC BAR CONSTRUCTION IN SYMMETRIC SPECTRA
}

\author{
IRAKLI PATCHKORIA AND STEFFEN SAGAVE \\ (Communicated by Michael A. Mandell)
}

\begin{abstract}
The cyclic bar construction in symmetric spectra and Bökstedt's original construction are two possible ways to define the topological Hochschild homology of a symmetric ring spectrum. In this short note we explain how to correct an error in Shipley's original comparison of these two approaches.
\end{abstract}

\section{INTRODUCTION}

When topological Hochschild homology was first introduced by Marcel Bökstedt in the unpublished manuscript [Bök85], a good point set level model for the smash product of spectra was not yet known, and THH was defined for functors with smash products. One can implement Bökstedt's definition for a symmetric ring spectrum $R$ by defining $\mathrm{THH}(R)$ to be the realization of the simplicial symmetric spectrum

$$
[k] \mapsto \mathrm{THH}_{k}(R)=\underset{\left(n_{0}, \ldots, n_{k}\right) \in \mathcal{I} \times k+1}{\operatorname{hocolim}} \Omega^{n_{0}+\cdots+n_{k}} L F_{0}\left(R_{n_{0}} \wedge \ldots \wedge R_{n_{k}}\right) .
$$

Here $\mathcal{I}$ is the category of finite sets and injections, $L$ is a level-fibrant replacement functor in symmetric spectra, and $F_{0}$ is the suspension spectrum functor. The functoriality of $\Omega^{n_{0}+\cdots+n_{k}} L F_{0}\left(R_{n_{0}} \wedge \ldots \wedge R_{n_{k}}\right)$ in the product category $\mathcal{I}^{\times k+1}$ comes from the structure maps of $R$, and the simplicial structure maps of $[k] \mapsto \mathrm{THH}_{k}(R)$ arise from the multiplication and unit of $R$; see Construction 2.2 below.

When viewing a symmetric ring spectrum $R$ as a monoid with respect to the smash product of symmetric spectra, one can also define its topological Hochschild homology as the realization of the cyclic bar construction $[k] \mapsto B_{k}^{\text {cy }}(R)=R^{\wedge k+1}$.

These two approaches are compared by Shipley in Shi00, Theorem 4.2.8]. The first step in her argument is to construct a chain of stable equivalences relating $B_{\bullet}^{\mathrm{cy}}(R)$ and the simplicial object

$$
[k] \mapsto \operatorname{hocolim}_{n \in \mathcal{I}} \Omega^{n} L F_{0}\left(R^{\wedge k+1}\right)_{n} .
$$

Next Shipley shows that there are canonical stable equivalences relating the simplicial degree $[k]$ parts of (1.1) and (1.2). However, it is erroneously stated in [Shi00, Theorem 4.2.8] that these maps form a morphism of simplicial objects. The problem is the compatibility with the last face map: The permutation of the $\mathcal{I}$-coordinates that goes into the last face map of the simplicial object (1.1) has no counterpart in the simplicial structure of (1.2). We make this precise in Example 2.3 below.

Received by the editors August 24, 2015 and, in revised form, November 17, 2015.

2010 Mathematics Subject Classification. Primary 55P43; Secondary 19D55.

The first author was supported by the Danish National Research Foundation through the Centre for Symmetry and Deformation (DNRF92). 
In Theorem 3.6 below we provide a comparison of these two definitions of $\mathrm{THH}$ that avoids this problem. Our strategy is to use a cofibrant replacement that allows us to replace homotopy colimits by colimits in the critical part of the argument.

1.1. Conventions. We assume familiarity with symmetric spectra and refer to HSS00 and Sch12 as useful references for this topic. We will often index spheres and the levels of symmetric spectra by finite sets $\mathbf{n}=\{1, \ldots, n\}$ rather than by natural numbers. This helps to keep track of permutation actions.

\section{TWO MODELS FOR THH}

Let $\mathcal{I}$ denote the category of finite sets $\mathbf{m}=\{1, \ldots, m\}, m \geq 0$, and injective maps. It is symmetric strict monoidal under the ordered concatenation of ordered sets $\mathbf{m} \sqcup \mathbf{n}=\mathbf{m}+\mathbf{n}$. The empty set $\mathbf{0}$ is the monoidal unit, and the block permutation $\tau_{(\mathbf{m}, \mathbf{n})}: \mathbf{m} \sqcup \mathbf{n} \rightarrow \mathbf{n} \sqcup \mathbf{m}$ provides the symmetry isomorphism for $\sqcup$.

As explained in DGM13, 2.2.2.1 and 4.2.1.1], applying the cyclic bar construction in the category of small categories (cat) to $\mathcal{I}$ provides a functor

$$
B_{\bullet}^{\text {cy }} \mathcal{I}: \Delta^{\text {op }} \rightarrow(\text { cat }), \quad[k] \mapsto \mathcal{I}^{\times k+1} .
$$

The simplicial face and degeneracy maps act by

$$
\begin{aligned}
& d_{i}\left(\mathbf{n}_{\mathbf{0}}, \ldots, \mathbf{n}_{\mathbf{k}}\right)=\left\{\begin{array}{ll}
\left(\mathbf{n}_{\mathbf{0}}, \ldots, \mathbf{n}_{\mathbf{i}} \sqcup \mathbf{n}_{\mathbf{i}+\mathbf{1}}, \ldots, \mathbf{n}_{\mathbf{k}}\right) & 0 \leq i<k \\
\left(\mathbf{n}_{\mathbf{k}} \sqcup \mathbf{n}_{\mathbf{0}}, \ldots, \mathbf{n}_{\mathbf{k}-\mathbf{1}}\right) & i=k
\end{array}\right. \text { and } \\
& s_{i}\left(\mathbf{n}_{\mathbf{0}}, \ldots, \mathbf{n}_{\mathbf{k}}\right)=\left(\mathbf{n}_{\mathbf{0}}, \ldots, \mathbf{n}_{\mathbf{i}}, \mathbf{0}, \mathbf{n}_{\mathbf{i}+\mathbf{1}}, \ldots, \mathbf{n}_{\mathbf{k}}\right) .
\end{aligned}
$$

Recall from [Tho79, 1.1 Definition] that the Grothendieck construction on a functor $F: \mathcal{C} \rightarrow$ (cat) is the category whose objects are the pairs $(C ; X)$ with $C \in \mathrm{Ob}(\mathcal{C})$ and $X \in \mathrm{Ob}(F(C))$. A morphism $(C ; X) \rightarrow(D ; Y)$ is a pair $(\alpha ; f)$ of morphisms $\alpha: C \rightarrow D$ in $\mathcal{C}$ and $f: F(\alpha)(X) \rightarrow Y$ in $F(D)$.

Definition 2.1. Let $B^{\text {cy }} \mathcal{I}$ be the Grothendieck construction of $B_{\bullet}^{\text {cy }} \mathcal{I}: \Delta^{\text {op }} \rightarrow$ (cat).

Let $\mathrm{Sp}^{\Sigma}$ be the category of symmetric spectra of simplicial sets and let $L=$ Sing $|-|: \mathrm{Sp}^{\Sigma} \rightarrow \mathrm{Sp}^{\Sigma}$ be the level fibrant replacement functor given by forming the singular complex of the geometric realization in each level. Let $\mathcal{S}_{*}$ be the category of pointed simplicial sets, and let $F_{0}: \mathcal{S}_{*} \rightarrow \mathrm{Sp}^{\Sigma}$ be the suspension spectrum functor.

The next construction is a reformulation of [Shi00, 4.2] and [DGM13, 4.2.2.3].

Construction 2.2. Let $R$ be an associative symmetric ring spectrum and let $M$ be an $R$-bimodule. Let

$$
\mathcal{D}(R ; M): B^{\mathrm{cy}} \mathcal{I} \rightarrow \mathrm{Sp}^{\Sigma}
$$

be the functor which is defined on objects by

$$
\left([k] ; \mathbf{n}_{\mathbf{0}}, \ldots, \mathbf{n}_{\mathbf{k}}\right) \mapsto \operatorname{Map}\left(S^{\mathbf{n}_{\mathbf{0}} \sqcup \ldots \sqcup \mathbf{n}_{\mathbf{k}}}, L F_{0}\left(M_{\mathbf{n}_{\mathbf{0}}} \wedge R_{\mathbf{n}_{\mathbf{1}}} \wedge \ldots \wedge R_{\mathbf{n}_{\mathbf{k}}}\right)\right) .
$$

The morphisms in $\mathcal{I}^{k+1}$ act via the symmetric group actions on the levels of $R$ and $M$ and the structure maps of the spectra $R$ and $M$ [DGM13, Definition 4.2.2.1]. The morphisms in $\Delta$ act as in DGM13, 4.2.2.3]. For example, the last face map gives rise to a morphism

$$
\left([k] ; \mathbf{n}_{\mathbf{0}}, \ldots, \mathbf{n}_{\mathbf{k}}\right) \rightarrow\left([k-1] ; \mathbf{n}_{\mathbf{k}} \sqcup \mathbf{n}_{\mathbf{0}}, \ldots, \mathbf{n}_{\mathbf{k}-\mathbf{1}}\right)
$$

in $B^{\text {cy }} \mathcal{I}$ which acts by using the symmetry isomorphism that moves $R_{\mathbf{n}_{\mathbf{k}}}$ to the front of the iterated smash product, the multiplication $R_{\mathbf{n}_{\mathbf{k}}} \wedge M_{\mathbf{n}_{\mathbf{0}}} \rightarrow M_{\mathbf{n}_{\mathbf{k}}} \sqcup \mathbf{n}_{\mathbf{0}}$, and 
the corresponding permutation of the sphere coordinates. The universal property of the Grothendieck construction and DGM13, Lemma 4.2.2.2] imply that this does indeed define a functor on $B^{\text {cy }} \mathcal{I}$. (The benefit of indexing $\mathcal{D}(R ; M)$ by $B^{\text {cy }} \mathcal{I}$ will become apparent in the next section.)

Writing $\mathcal{D}_{k}(R ; M)=\mathcal{D}(R ; M)([k] ;-): \mathcal{I}^{\times k+1} \rightarrow \mathrm{Sp}^{\Sigma}$, we get a simplicial object in symmetric spectra

$$
[k] \mapsto \operatorname{hocolim}_{\mathcal{I} \times k+1} \mathcal{D}_{k}(R ; M)=: \operatorname{THH}_{k}(R ; M)
$$

where $\alpha:[k] \rightarrow[l]$ in $\Delta$ acts by

$\operatorname{hocolim}_{\mathcal{I}^{\times l+1}} \mathcal{D}_{l}(R ; M) \rightarrow \operatorname{hocolim}_{\mathcal{I}^{\times} \times+1} \mathcal{D}_{k}(R ; M) \circ \alpha^{*} \rightarrow \operatorname{hocolim}_{\mathcal{I}^{\times k+1}} \mathcal{D}_{k}(R ; M)$.

Here the first map is induced by the functoriality of $\mathcal{D}(R ; M)$ in $B^{\mathrm{cy}} \mathcal{I}$. As discussed in the introduction, the realization of THH. $(R ; M)$ is Bökstedt's model for THH.

Now let

$$
B_{\bullet}^{\mathrm{cy}}(R ; M): \Delta^{\mathrm{op}} \rightarrow \mathrm{Sp}_{,}^{\Sigma} \quad[k] \mapsto M \wedge R^{\wedge k},
$$

be the cyclic bar construction in $\left(\mathrm{Sp}^{\Sigma}, \wedge, \mathbb{S}\right)$; see e.g. [DGM13, 4.2.1.1]. Let

$$
\Omega_{\mathrm{Sp}}^{\mathcal{I}}: \mathrm{Sp}^{\Sigma} \rightarrow\left(\mathrm{Sp}^{\Sigma}\right)^{\mathcal{I}}, \quad\left(\Omega_{\mathrm{Sp}}^{\mathcal{I}}\right)(X)(\mathbf{m})=\operatorname{Map}\left(S^{\mathbf{m}}, L F_{0} X_{\mathbf{m}}\right),
$$

be the functor where isomorphisms $\mathbf{m} \rightarrow \mathbf{m}$ in $\mathcal{I}$ act by conjugation and inclusions $\mathbf{m}-\mathbf{1} \rightarrow \mathbf{m}$ act via the structure map of $X$. Then hocolim ${ }_{\mathcal{I}} \Omega_{\mathrm{Sp}}^{\mathcal{I}}: \mathrm{Sp}^{\Sigma} \rightarrow \mathrm{Sp}^{\Sigma}$ is Shipley's detection functor [Shi00, Definition 3.1.1]. It is shown in the first part of the proof of [Shi00, Theorem 4.2.8] that there is a zig-zag of degreewise stable equivalences of simplicial objects in symmetric spectra relating hocolim $\Omega_{\mathcal{I}} \Omega_{\mathrm{Sp}}^{\mathcal{I}} B_{\bullet}^{\mathrm{cy}}(R ; M)$ and $B_{\bullet}^{\mathrm{cy}}(R ; M)$. To relate the former object to $\mathrm{THH}_{\bullet}(R ; M)$, we note that there is a canonical map

$$
M_{\mathbf{n}_{0}} \wedge R_{\mathbf{n}_{1}} \wedge \ldots \wedge R_{\mathbf{n}_{\mathbf{k}}} \rightarrow(M \wedge R \wedge \ldots \wedge R)_{\mathbf{n}_{\mathbf{0}} \sqcup \ldots \sqcup \mathbf{n}_{\mathbf{k}}} .
$$

(The map arises for example from identifying $(X \wedge Y)_{n}$ for symmetric spectra $X$

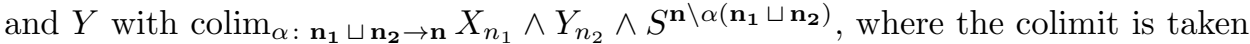
over the comma category $-\sqcup-\downarrow \mathbf{n}$.) Writing $\mu_{k+1}: \mathcal{I}^{\times k+1} \rightarrow \mathcal{I}$ for the iterated concatenation, the map (2.2) induces a morphism of symmetric spectra

$$
\begin{aligned}
& \mathrm{THH}_{k}(R ; M)=\operatorname{hocolim}_{\mathcal{I}^{\times k+1}} \mathcal{D}_{k}(R ; M) \\
& \quad \rightarrow \operatorname{hocolim}_{\mathcal{I}^{\times k+1}} \mu_{k+1}^{*} \Omega_{\mathrm{Sp}}^{\mathcal{I}}\left(M \wedge R^{\wedge k}\right) \rightarrow \operatorname{hocolim}_{\mathcal{I}} \Omega_{\mathrm{Sp}}^{\mathcal{I}}\left(M \wedge R^{\wedge k}\right) .
\end{aligned}
$$

The problem with the proof of [Shi00, Theorem 4.2.8] is that this map fails to provide a map of simplicial objects:

Example 2.3. We examine how the comparison maps in simplicial levels 0 and 1 interact with $d_{1}$. To simplify the exposition, we here ignore the suspension spectrum functor and the level fibrant replacement. Let $f: S^{\mathbf{n}_{\mathbf{0}}} \sqcup \mathbf{n}_{\mathbf{1}} \rightarrow M_{\mathbf{n}_{\mathbf{0}}} \wedge R_{\mathbf{n}_{1}}$ represent a 0 -simplex in hocolim $\operatorname{I}_{\mathcal{I} \times 2} \mathcal{D}_{1}(R ; M)$. First applying the map (2.3) and then the simplicial structure map $d_{1}$ of $B_{\bullet}^{\text {cy }}(R ; M)$ to $f$ amounts to forming the composite

$$
S^{\mathbf{n}_{\mathbf{0}} \sqcup \mathbf{n}_{1}} \stackrel{f}{\rightarrow} M_{\mathbf{n}_{\mathbf{0}}} \wedge R_{\mathbf{n}_{\mathbf{1}}} \rightarrow(M \wedge R)_{\mathbf{n}_{\mathbf{0}}} \sqcup \mathbf{n}_{\mathbf{1}} \stackrel{\tau}{\rightarrow}(R \wedge M)_{\mathbf{n}_{\mathbf{0}} \sqcup \mathbf{n}_{\mathbf{1}}} \stackrel{\mu}{\rightarrow} M_{\mathbf{n}_{\mathbf{0}}} \sqcup \mathbf{n}_{\mathbf{1}} .
$$

Applying first $d_{1}$ and then the map (2.3) sends $f$ to the composite

$$
S^{\mathbf{n}_{1} \sqcup \mathbf{n}_{\mathbf{0}}} \stackrel{\tau_{\left(\mathbf{n}_{1}, \mathbf{n}_{\mathbf{0}}\right)}^{\longrightarrow}}{\rightarrow} S^{\mathbf{n}_{\mathbf{0}} \sqcup \mathbf{n}_{1}} \stackrel{f}{\rightarrow} M_{\mathbf{n}_{\mathbf{0}}} \wedge R_{\mathbf{n}_{\mathbf{1}}} \stackrel{\tau}{\rightarrow} R_{\mathbf{n}_{\mathbf{1}}} \wedge M_{\mathbf{n}_{\mathbf{0}}} \stackrel{\mu}{\rightarrow} M_{\mathbf{n}_{\mathbf{1}}} \sqcup \mathbf{n}_{\mathbf{0}} .
$$


However, inspecting the commutative diagram

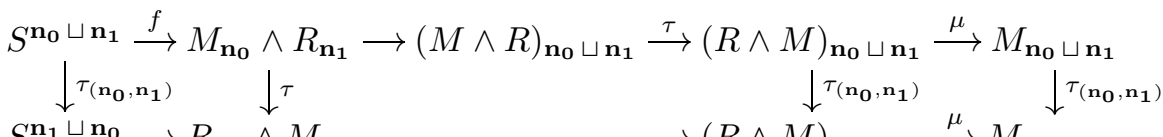

$$
\begin{aligned}
& S^{\mathbf{n}_{1}} \sqcup \mathbf{n}_{\mathbf{0}} \longrightarrow R_{\mathbf{n}_{\mathbf{1}}} \wedge M_{\mathbf{n}_{\mathbf{0}}} \longrightarrow(R \wedge M)_{\mathbf{n}_{\mathbf{1}} \sqcup \mathbf{n}_{\mathbf{0}}} \stackrel{\mu}{\longrightarrow} M_{\mathbf{n}_{\mathbf{1}}} \sqcup \mathbf{n}_{\mathbf{0}},
\end{aligned}
$$

we deduce that the two maps (2.4) and (2.5) differ by the conjugation action of the block permutation $\tau_{\left(\mathbf{n}_{\mathbf{0}}, \mathbf{n}_{1}\right)}: \mathbf{n}_{\mathbf{0}} \sqcup \mathbf{n}_{\mathbf{1}} \rightarrow \mathbf{n}_{\mathbf{1}} \sqcup \mathbf{n}_{\mathbf{0}}$. In fact, this is already indicated by the order of $\mathbf{n}_{\mathbf{0}}$ and $\mathbf{n}_{\mathbf{1}}$. Hence the points in hocolim $\Omega_{\mathcal{I}} \Omega_{\mathrm{Sp}}^{\mathcal{I}}\left(M \wedge R^{\wedge k}\right)$ represented by the two maps (2.4) and (2.5) do not coincide in general. Instead, they are only connected by the 1-simplex represented by the morphism $\tau_{\left(\mathbf{n}_{\mathbf{0}}, \mathbf{n}_{1}\right)}$ in $\mathcal{I}$.

This shows that the maps (2.3) fail to be compatible with the simplicial structure maps and do not induce a morphism on the realization.

\section{Diagrams indeXed By the CYCLIC BAR CONSTRUCTION ON $\mathcal{I}$}

We now return to the setup of Definition 2.1. Let us for a moment view the iterated concatenation in $\mathcal{I}$ as a functor

$$
\mu_{k+1}: \mathcal{I}^{\times k+1} \rightarrow \Delta^{\mathrm{op}} \times \mathcal{I}, \quad\left(\mathbf{n}_{\mathbf{0}}, \ldots, \mathbf{n}_{\mathbf{k}}\right) \mapsto\left([k], \mathbf{n}_{\mathbf{0}} \sqcup \ldots \sqcup \mathbf{n}_{\mathbf{k}}\right) .
$$

We claim that each $\alpha:[k] \rightarrow[l]$ in $\Delta$ induces a natural transformation

$$
\bar{\alpha}: \mu_{l+1} \Rightarrow \mu_{k+1} \circ \alpha^{*}
$$

such that for $\beta:[l] \rightarrow[m]$, the following composition rule is satisfied:

$$
\overline{\beta \alpha}=\left(\bar{\alpha} \beta^{*}\right)(\bar{\beta}): \mu_{m+1} \Rightarrow \mu_{k+1} \circ \alpha^{*} \circ \beta^{*}=\mu_{k+1} \circ(\beta \alpha)^{*} .
$$

To define $\bar{\alpha}$, we set $\bar{\alpha}=(\alpha$, id) if $\alpha$ is a degeneracy map or a face map that is not equal to the last face map, and $\bar{\alpha}=\left(\alpha, \tau_{\left(\mathbf{n}_{\mathbf{0}} \sqcup \ldots \sqcup \mathbf{n}_{\mathbf{k}-\mathbf{1}}, \mathbf{n}_{\mathbf{k}}\right)}\right)$ if $\alpha$ is the last face map. Writing a general $\alpha$ as a composite of face and degeneracy maps, we can define $\bar{\alpha}$ by the above composition formula. This is well defined since our definition of $\bar{\alpha}$ for the face and degeneracy maps is compatible with the simplicial identities. By the universal property of the Grothendieck construction [Tho79, 1.3.1 Proposition], we thus get a functor

$$
\mu^{\mathrm{tw}}: B^{\mathrm{cy}} \mathcal{I} \rightarrow \Delta^{\mathrm{op}} \times \mathcal{I}
$$

sending $\left([k] ; \mathbf{n}_{\mathbf{0}}, \ldots, \mathbf{n}_{\mathbf{k}}\right)$ to $\left([k], \mathbf{n}_{\mathbf{0}} \sqcup \ldots \sqcup \mathbf{n}_{\mathbf{k}}\right)$.

Definition 3.1. Let $E: \Delta^{\mathrm{op}} \rightarrow\left(\mathrm{Sp}^{\Sigma}\right)^{\mathcal{I}}$ be a simplicial object in $\mathcal{I}$-diagrams of symmetric spectra. Viewing it as a functor $E: \Delta^{\mathrm{op}} \times \mathcal{I} \rightarrow \mathrm{Sp}^{\Sigma}$, we let

$$
E^{\mathrm{tw}}: B^{\mathrm{cy}} \mathcal{I} \rightarrow \mathrm{Sp}^{\Sigma}
$$

be the composite $E \circ \mu^{\mathrm{tw}}$ of $E$ with the functor (3.1).

We note that for $E: \Delta^{\mathrm{op}} \rightarrow\left(\mathrm{Sp}^{\Sigma}\right)^{\mathcal{I}}$, there is a canonical map

$$
\underset{\mathcal{I} \times k+1}{\operatorname{hocolim}} E^{\mathrm{tw}}([k] ;-) \stackrel{\cong}{\rightarrow} \underset{\mathcal{I} \times k+1}{\operatorname{hocolim}} \mu_{k+1}^{*} E([k],-) \rightarrow \underset{\mathcal{I}}{\operatorname{hocolim}} E([k],-) .
$$

Analogous to Example 2.3, the maps (3.2) do in general fail to be compatible with the last face map $d_{k}$ and thus do not assemble to a map of simplicial objects. However, composing with the map from the homotopy colimit to the colimit, this can be resolved. 
Lemma 3.2. The morphisms (3.2) become compatible with the simplicial structure maps after composing them with the canonical map $\operatorname{hocolim}_{\mathcal{I}} \rightarrow \operatorname{colim}_{\mathcal{I}}$.

Proof. Since the map from the homotopy colimit to the colimit is natural with respect to the change of the index category, it is sufficient to show that $\alpha:[k] \rightarrow[l]$ in $\Delta$ induces a commutative diagram

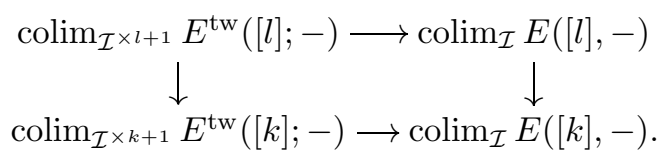

This is easy to verify for the degeneracy maps and all face maps but the last one. Let $\delta^{l}:[l-1] \rightarrow[l]$ be the last face map in $\Delta$, and let $x \in E^{\mathrm{tw}}\left([l] ; \mathbf{n}_{\mathbf{0}}, \ldots, \mathbf{n}_{\mathbf{l}}\right)$ represent a simplex in one of the levels of the spectrum $\operatorname{colim}_{\mathcal{I}^{\times l+1}} E^{\mathrm{tw}}([l] ;-)$. Then the composite through the upper right hand corner sends $x$ to the simplex represented by $\left(\delta^{l}\right)^{*}(x) \in E\left([l-1], \mathbf{n}_{\mathbf{0}} \sqcup \ldots \sqcup \mathbf{n}_{\mathbf{l}}\right)$, while the other composite sends it to the simplex represented by

$$
\left(\tau_{\left(\mathbf{n}_{\mathbf{0}} \sqcup \ldots \sqcup \mathbf{n}_{\mathbf{1}-\mathbf{1}}, \mathbf{n}_{\mathbf{1}}\right)}\right)_{*}\left(\left(\delta^{l}\right)^{*}(x)\right) \in E\left([l-1], \mathbf{n}_{\mathbf{l}} \sqcup \mathbf{n}_{\mathbf{0}} \sqcup \ldots \sqcup \mathbf{n}_{\mathbf{l}-\mathbf{1}}\right) .
$$

These represent the same simplex in the colimit.

We need some preparation to apply the lemma in a useful way.

Definition 3.3. Let $R$ be an associative symmetric ring spectrum and let $M$ be an $R$-bimodule. Then the twisted cyclic bar construction is the $B^{\text {cy }} \mathcal{I}$-diagram

$$
B^{\mathrm{cy}}(R ; M)^{\mathrm{tw}}=\Omega_{\mathrm{Sp}}^{\mathcal{I}}\left(B_{\bullet}^{\mathrm{cy}}(R ; M)\right)^{\mathrm{tw}}: B^{\mathrm{cy}} \mathcal{I} \rightarrow \mathrm{Sp}^{\Sigma}
$$

where $\Omega_{\mathrm{Sp}}^{\mathcal{I}}$ and $B_{\bullet}^{\mathrm{cy}}(R ; M)$ are as in the last section.

Recall that a symmetric spectrum $X$ is semistable if it admits a $\pi_{*}$-isomorphism to a symmetric $\Omega$-spectrum [HSS00, 5.6], and that it is flat if it is $S$-cofibrant, i.e., cofibrant in the $S$-model structure developed in Shi04. We call a symmetric ring spectrum flat if its underlying symmetric spectrum is flat.

Proposition 3.4. The canonical maps to the smash product (2.2) induce a natural transformation of $B^{\text {cy }} \mathcal{I}$-diagrams $\mathcal{D}(R ; M) \rightarrow B^{\mathrm{cy}}(R ; M)^{\mathrm{tw}}$. Fixing a simplicial degree $[k]$, the induced map

$$
\operatorname{THH}_{k}(R ; M)=\operatorname{hocolim}_{\mathcal{I}^{\times k+1}} \mathcal{D}_{k}(R ; M) \rightarrow \operatorname{hocolim}_{\mathcal{I}^{\times k+1}} B^{\mathrm{cy}}(R ; M)^{\mathrm{tw}}([k] ;-)
$$

is a stable equivalence if $R$ is flat and $R$ and $M$ are semistable.

Proof. It follows from the definitions that there is an induced map. The argument given in the proof of Shi00, Theorem 4.2.8], which is in turn based on Shi00, Proposition 4.2.3], shows that the composite of the map in the statement of the proposition with the map (3.2) for $E=\Omega_{\mathrm{Sp}}^{\mathcal{I}}\left(B_{\bullet}^{\mathrm{cy}}(R ; M)\right)$ is a stable equivalence. Hence it is enough to show that

$$
\operatorname{hocolim}_{\mathcal{I} \times k+1} \mu_{k+1}^{*}\left(\Omega_{\mathrm{Sp}}^{\mathcal{I}}\left(B_{k}^{\text {cy }}(R ; M)\right)\right) \rightarrow \operatorname{hocolim}_{\mathcal{I}} \Omega_{\mathrm{Sp}}^{\mathcal{I}}\left(B_{k}^{\text {cy }}(R ; M)\right)
$$

is a stable equivalence. This follows from Lemma 3.10 and Lemma 3.12 below since by [Sch08, 4.10 Theorem], our assumptions on $R$ and $M$ imply that $B_{k}^{\text {cy }}(R ; M)$ is semistable. 
To apply Lemma 3.2 to the cyclic bar construction, we employ the projective model structure on the diagram category $\left(\mathrm{Sp}^{\Sigma}\right)^{\Delta^{\mathrm{op}} \times \mathcal{I}}=\left(\left(\mathrm{Sp}^{\Sigma}\right)^{\Delta^{\mathrm{op}}}\right)^{\mathcal{I}}$. This is the model structure where a natural transformation $f: X \rightarrow Y$ of $\Delta^{\text {op }} \times \mathcal{I}$-diagrams of symmetric spectra is a weak equivalence or fibration if $f([k], \mathbf{m})$ is a weak equivalence or fibration in the absolute projective stable model structure on $\mathrm{Sp}^{\Sigma}$ for all objects $([k], \mathbf{m})$ of $\Delta^{\mathrm{op}} \times \mathcal{I}$. Let

$$
C \stackrel{\sim}{\longrightarrow} \Omega_{\mathrm{Sp}}^{\mathcal{I}}\left(B_{\bullet}^{\mathrm{cy}}(R ; M)\right)
$$

be a cofibrant resolution in this model structure. Inspecting the generating cofibrations of the projective model structure on $\left(\mathrm{Sp}^{\Sigma}\right)^{\Delta^{\mathrm{op}} \times \mathcal{I}}$, it follows that for each $[k]$, the map $C([k],-) \stackrel{\sim}{\longrightarrow} \Omega_{\mathrm{Sp}}^{\mathcal{I}}\left(B_{k}^{\text {cy }}(R ; M)\right)$ is a cofibrant replacement in $\left(\mathrm{Sp}^{\Sigma}\right)^{\mathcal{I}}$.

Proposition 3.5. The cofibrant replacement and the natural map from the homotopy colimit to the colimit induce a zig-zag of stable equivalences

$$
\begin{aligned}
& \operatorname{hocolim}_{\mathcal{I} \times k+1} B^{\mathrm{cy}}(R ; M)^{\mathrm{tw}}([k] ;-) \stackrel{\sim}{\leftarrow} \operatorname{hocolim}_{\mathcal{I} \times k+1} C^{\mathrm{tw}}([k] ;-) \\
& \stackrel{\sim}{\longrightarrow} \operatorname{colim}_{\mathcal{I}} C([k],-) \stackrel{\sim}{\leftarrow} \operatorname{hocolim}_{\mathcal{I}} C([k],-) \stackrel{\sim}{\longrightarrow} \operatorname{hocolim}_{\mathcal{I}} \Omega_{\mathrm{Sp}}^{\mathcal{I}}\left(B_{k}^{\mathrm{cy}}(R ; M)\right)
\end{aligned}
$$

that is compatible with the simplicial structure maps.

We prove the proposition at the end of the section.

Theorem 3.6. Let $R$ be a flat symmetric ring spectrum, let $M$ be an $R$-bimodule spectrum, and assume that $R$ and $M$ are semistable. Then there is a zig-zag of degreewise stable equivalences of simplicial objects relating $B_{\bullet}^{\mathrm{cy}}(R ; M)$ and $\mathrm{THH} \bullet(R ; M)$. It induces a chain of stable equivalences after realization.

Proof. This follows by combining Propositions 3.4 and 3.5 with the chain of degreewise stable equivalences relating $B_{\bullet}^{\mathrm{cy}}(R ; M)$ and hocolim ${ }_{\mathcal{I}} \Omega_{\mathrm{Sp}}^{\mathcal{I}}\left(B_{\bullet}^{\mathrm{cy}}(R ; M)\right)$ from the proof of [Shi00, Theorem 4.2.8].

Remark 3.7. One can use the argument outlined in [Shi00, Remark 4.2.10] to get to a more general statement that avoids the semistability assumption in the previous theorem.

Remark 3.8. When $M=R$, both $B_{\bullet}^{\text {cy }}(R ; M)$ and $\mathrm{THH}_{\bullet}(R ; M)$ are cyclic objects, i.e., they extend to functors $\Lambda^{\mathrm{op}} \rightarrow \mathrm{Sp}^{\Sigma}$ on Connes' cyclic category $\Lambda$. Replacing $\Delta$ in our constructions by $\Lambda$ leads to a chain of stable equivalences relating these cyclic objects and therefore to a cyclic version of Theorem 3.6. After realization of the cyclic objects involved, we thus obtain a chain of stable equivalences of symmetric spectra with $S^{1}$-action relating $B^{\mathrm{cy}}(R)=\left|B_{\bullet}^{\mathrm{cy}}(R ; R)\right|$ and $\operatorname{THH}(R)=$ $\left|\mathrm{THH}_{\bullet}(R ; R)\right|$.

In view of the cyclotomic structure on the cyclic bar construction (of an orthogonal ring spectrum) recently established by Angeltveit et al. $\left[\mathrm{ABG}^{+} 15\right]$, one may ask if this zig-zag of stable equivalences induces a zig-zag of stable equivalences relating the resulting topological cyclic homology spectra. We don't have evidence that this follows directly from the present result. In fact, already the zig-zag of stable equivalences $B^{\mathrm{cy}}(R) \simeq \operatorname{hocolim}_{\mathcal{I}} \Omega_{\mathrm{Sp}}^{\mathcal{I}}\left(B^{\mathrm{cy}}(R)\right)$ from the proof of [Shi00, Theorem 4.2.8] does not appear to be well behaved with the passage to fixed points. 
3.9. Semistability results. An $\mathcal{I}$-space $X$ is a functor $X: \mathcal{I} \rightarrow \mathcal{S}_{*}$ from $\mathcal{I}$ to based simplicial sets. Let $\mathcal{N} \subset \mathcal{I}$ be the subcategory given by the standard inclusions. A map of $\mathcal{I}$-spaces $X \rightarrow Y$ is an $\mathcal{N}$-equivalence if the induced map of based homotopy $\operatorname{colimits}_{\text {hocolim }} X \rightarrow \operatorname{hocolim}_{\mathcal{N}} Y$ is a weak equivalence of spaces. An $\mathcal{I}$-space $X$ is semistable if there is an $\mathcal{N}$-equivalence $X \rightarrow Y$ with $Y$ homotopy constant, i.e., every $\alpha: \mathbf{m} \rightarrow \mathbf{n}$ induces a weak equivalence $\alpha_{*}: Y(\mathbf{m}) \rightarrow Y(\mathbf{n})$. This notion of semistability is studied in [SS13, 2.5] in the case of unbased $\mathcal{I}$-spaces.

Lemma 3.10. Let $X: \mathcal{I} \rightarrow \mathcal{S}_{*}$ be a semistable $\mathcal{I}$-space. Then the canonical map

$$
\operatorname{hocolim}_{\mathcal{I} \times k} \mu_{k}^{*}(X) \rightarrow \operatorname{hocolim}_{\mathcal{I}} X
$$

is a weak equivalence.

Proof. Suppose first that $X$ is homotopy constant. Then the canonical maps

$$
X\left(\mathbf{n}_{\mathbf{1}} \sqcup \ldots \sqcup \mathbf{n}_{\mathbf{k}}\right) \rightarrow \operatorname{hocolim}_{\mathcal{I}^{\times k}} \mu_{k}^{*}(X) \quad \text { and } \quad X\left(\mathbf{n}_{\mathbf{1}} \sqcup \ldots \sqcup \mathbf{n}_{\mathbf{k}}\right) \rightarrow \operatorname{hocolim}_{\mathcal{I}} X
$$

are weak equivalences since the classifying spaces of $\mathcal{I}$ and $\mathcal{I}^{\times k}$ are contractible; see e.g. Dug01, Proposition 5.4]. This implies the result for a homotopy constant $X$. For a semistable $X$, it is now sufficient to show that an $\mathcal{N}$-equivalence $X \rightarrow Y$ induces weak equivalences

$\operatorname{hocolim}_{\mathcal{I}} X \rightarrow \operatorname{hocolim}_{\mathcal{I}} Y$ and $\operatorname{hocolim}_{\mathcal{I}^{\times k}} \mu_{k}^{*}(X) \rightarrow \operatorname{hocolim}_{\mathcal{I}^{\times k}} \mu_{k}^{*}(Y)$.

For the first map this follows from [Shi00, Proposition 2.2.9]. The claim about the second map follows since there is a weak equivalence

$$
\underset{\mathcal{I} \times k}{\operatorname{hocolim}} \mu_{k}^{*}(X) \stackrel{\sim}{\rightarrow} \underset{\left(\mathbf{n}_{\mathbf{1}}, \ldots, \mathbf{n}_{\mathbf{k}-\mathbf{1}}\right) \in \mathcal{I} \times k-1}{\operatorname{hocolim}} \underset{\mathbf{n}_{\mathbf{k}} \in \mathcal{I}}{\operatorname{hocolim}} X\left(\left(\mathbf{n}_{\mathbf{1}} \sqcup \ldots \sqcup \mathbf{n}_{\mathbf{k}-\mathbf{1}}\right) \sqcup \mathbf{n}_{\mathbf{k}}\right)
$$

and restriction along $\left(\mathbf{n}_{\mathbf{1}} \sqcup \ldots \sqcup \mathbf{n}_{\mathbf{k}-\mathbf{1}}\right) \sqcup-: \mathcal{I} \rightarrow \mathcal{I}$ preserves $\mathcal{N}$-equivalences by a cofinality argument.

Remark 3.11. Since the classifying space of the undercategory $\mathbf{1} \downarrow \mu_{2}$ has two path components, the functor $\mu_{k}$ is in general not homotopy cofinal, and the last lemma does not hold without the semistability hypothesis.

Lemma 3.12. Let $E$ be a semistable symmetric spectrum. Then $\Omega_{\mathrm{Sp}}^{\mathcal{I}}(E)$ is a semistable $\mathcal{I}$-space in every spectrum degree.

Proof. Let $E \rightarrow F$ be a $\pi_{*}$-isomorphism to a symmetric $\Omega$-spectrum $F$. Then in spectrum level 0 , the induced map $\Omega_{\mathrm{Sp}}^{\mathcal{I}}(E) \rightarrow \Omega_{\mathrm{Sp}}^{\mathcal{I}}(F)$ is an $\mathcal{N}$-equivalence to a homotopy constant $\mathcal{I}$-space. The $\mathcal{I}$-space in spectrum level $k>0$ of $\Omega_{\mathrm{Sp}}^{\mathcal{I}}(E)$ is isomorphic to the $\mathcal{I}$-space in spectrum level 0 of the $\mathcal{I}$-symmetric spectrum $\Omega_{\mathrm{Sp}}^{\mathcal{I}}\left(S^{k} \wedge E\right)$ associated with the symmetric spectrum $S^{k} \wedge E$. Since $S^{k} \wedge E$ is semistable if $E$ is [Sch08, 4.6 Example], the level 0 case implies the general case.

Proof of Proposition 3.5. The compatibility with the simplicial structure maps follows from Lemma 3.2 It is clear that the first and the last map are stable equivalences. The third map is a stable equivalence because $C([k],-)$ is cofibrant in $\left(\mathrm{Sp}^{\Sigma}\right)^{\mathcal{I}}$. Using once more that hocolim ${ }_{\mathcal{I}} C([k],-) \rightarrow \operatorname{colim}_{\mathcal{I}} C([k],-)$ is a stable equivalence reduces the claim about the second map to showing that $\operatorname{hocolim}_{\mathcal{I} \times k+1} C^{\mathrm{tw}}([k] ;-) \rightarrow \operatorname{hocolim}_{\mathcal{I}} C([k],-)$ is a stable equivalence. In view of Lemma 3.10, it is sufficient to show that $C([k],-)$ is semistable as an $\mathcal{I}$-space in every spectrum degree. Since the cofibrant replacement (3.3) is an objectwise level acyclic fibration of symmetric spectra, this follows from Lemma 3.12 since our 
assumptions on $R$ and $M$ imply that $B_{k}^{\text {cy }}(R ; M)$ is semistable; see [Sch08, 4.10 Theorem].

\section{ACKNOWLEDGMEnTS}

After we first discovered the error in Shi00, Theorem 4.2.8] and later found the present workaround, Brooke Shipley encouraged us to prepare this note and make it available. We thank Stefan Schwede for comments on an earlier version of this note. We also thank the referees for useful comments.

\section{REFERENCES}

$\left[\mathrm{ABG}^{+} 15\right]$ V. Angeltveit, A. Blumberg, T. Gerhardt, M. Hill, T. Lawson, and M. Mandell, Topological cyclic homology via the norm, 2015, arXiv:1401.5001v2

[Bök85] M. Bökstedt, Topological Hochschild homology, Preprint, Bielefeld, 1985.

[DGM13] Bjørn Ian Dundas, Thomas G. Goodwillie, and Randy McCarthy, The local structure of algebraic K-theory, Algebra and Applications, vol. 18, Springer-Verlag London, Ltd., London, 2013. MR3013261

[Dug01] Daniel Dugger, Replacing model categories with simplicial ones, Trans. Amer. Math. Soc. 353 (2001), no. 12, 5003-5027 (electronic), DOI 10.1090/S0002-9947-01-02661-7. MR.1852091 (2002f:55043)

[HSS00] Mark Hovey, Brooke Shipley, and Jeff Smith, Symmetric spectra, J. Amer. Math. Soc. 13 (2000), no. 1, 149-208, DOI 10.1090/S0894-0347-99-00320-3. MR.1695653 (2000h:55016)

[Sch08] Stefan Schwede, On the homotopy groups of symmetric spectra, Geom. Topol. 12 (2008), no. 3, 1313-1344, DOI 10.2140/gt.2008.12.1313. MR2421129 (2009c:55006)

[Sch12] Stefan Schwede, Symmetric spectra, 2012, Book project, available at the author's home page.

[Shi00] Brooke Shipley, Symmetric spectra and topological Hochschild homology, K-Theory 19 (2000), no. 2, 155-183, DOI 10.1023/A:1007892801533. MR.1740756 (2001h:55010)

[Shi04] Brooke Shipley, A convenient model category for commutative ring spectra, Homotopy theory: relations with algebraic geometry, group cohomology, and algebraic $K$-theory, Contemp. Math., vol. 346, Amer. Math. Soc., Providence, RI, 2004, pp. 473-483, DOI 10.1090/conm/346/06300. MR2066511(2005d:55014)

[SS13] Steffen Sagave and Christian Schlichtkrull, Group completion and units in $\mathcal{I}$-spaces, Algebr. Geom. Topol. 13 (2013), no. 2, 625-686, DOI 10.2140/agt.2013.13.625. MR.3044590

[Tho79] R. W. Thomason, Homotopy colimits in the category of small categories, Math. Proc. Cambridge Philos. Soc. 85 (1979), no. 1, 91-109, DOI 10.1017/S0305004100055535. MR.510404 (80b:18015)

Department of Mathematical Sciences, University of Copenhagen, Universitetsparken 5, 2100 Copenhagen $\varnothing$, Denmark

E-mail address: irakli.p@math.ku.dk

Radboud University Nijmegen, IMAPP, P.O. Box 9010, 6500 GL Nijmegen, The NetherLANDS

E-mail address: s.sagave@math.ru.nl 